\title{
Personalized Recommendation Algorithm of E-commerce based on Cloud Computing
}

\author{
Chongming Xu, Jinyan Zhang \\ Department of Educational Administration, Qingdao Huanghai University, Qingdao, \\ Shandong, China
}

\begin{abstract}
The project-based collaborative filtering algorithm and matrix decomposition based ALS collaborative filtering algorithm in the personalized recommendation of e-commerce are analyzed deeply in the paper, the principles of parallelization work are discussed, and the parallelization processes of project-based collaborative filtering algorithm improvement and matrix decomposition-based ALS collaborative filtering algorithm are offered via using MapReduce parallel programming model.
\end{abstract}

Keywords: E-commerce, Recommendation Algorithm, Cloud Computing

\section{Introduction}

As a platform providing the network shopping for customers, e-commerce sites hope to recommend the goods that the customers may be interested more accurately via using the recommendation technology with a better efficiency to improve the overall sales and profits of the sites. One main feature of personalized recommendation system is that it can make different personalized recommendation actively for customers via the their user data collected by the e-commerce sites and according to their historical behaviors[1], such as buying record, interest preference and others, and realize the one-to-one personalized service. Moreover, the goods the e-commerce sites recommend for users are updated in real time, and they are changed according to the goods type in the system or customer's preferences. In this way, it not only greatly improves the convenience and effectiveness of e-commerce trade, but also improves the merchant's service quality[2].

The collaborative filtering recommendation algorithm is mainly used in the personalized recommendation algorithm of e-commerce based on cloud computing researched in the paper. The traditional collaborative filtering algorithm has a wide range of researches, while there are some disadvantages in the recommendation speed and quality[3]. Two kinds of improved recommendation algorithms based on traditional collaborative filtering are adopted, one is the project-based collaborative filtering recommendation algorithm[4], and the other is matrix decomposition-based ALS recommendation algorithm. These two algorithms improve the sparse matrix problem in the traditional recommendation algorithm to certain degree. These two kinds of basic algorithms are introduced in the paper firstly in details, the improve principle and skill are introduced, and the improved algorithm is offered. 


\section{Project-based collaborative filtering recommendation algorithm}

Different from the traditional user-based collaborative filtering recommendation algorithm, the project-based collaborative filtering recommendation algorithm alleviates the huge calculation pressure problem caused by the increased user amount. For the project-based collaborative filtering algorithm, we shall firstly establish the customer's recommendation behavior on the goods, including browsing the web, stay purchasing goods, grading the bought goods, etc. The user's scoring on the goods is calculated according to the in-advanced set weight, the similarity between any two goods can be obtained according to the existing user's scoring on the goods, further the most similar list of each goods can be found, user's predictive score on the goods without scoring can be calculated, and user can be recommended.

The application of project-based collaborative filtering algorithm process on the e-commerce mainly includes three steps: collecting user's historical behavior data in e-commerce web and displaying the relationship between user and goods, calculating the similarity among goods, and predicting the scoring of the goods.

\section{Parallelization of project-based collaborative filtering recommendation algorithm under cloud platform}

The most important two steps of project-based collaborative filtering algorithm are seeking the similarity of scored project according to the project user's scoring matrix via certain similarity calculation method, and then predicting the project that the target user does not score according to the obtained similarity.

According to the above analysis, the first step is analyzed firstly by the personalized recommendation algorithm of e-commerce based on cloud computing, and the similarity of two goods can be obtained. The parallelization process can be analyzed according to the definition of MapReduce programming model. If the similarity of any two goods is required to obtain, we shall firstly know the common users' scoring on these two goods. For example, if the similarity of goods 1 and goods 2 is required, we need to know the common scores of these two goods from all users scored the goods, namely such as < (item 1, item 2), (user 1's score on item 1, user 1's score on item 2)> form user- goods mapping collection. If collecting the goods score and user, we need to obtain user's all scored goods, namely < user 1, (item 1, item 2, item 3,...)>. It is easier to obtain the similar key-value via MapReduce operation conversion, therefore, two MapReduce operations can be used to complete the above process. In the following, the operation among different users can be processed in parallel when making the predictive scoring for goods, and MapReduce operation can be used for processing. The similarity calculation and predictive scoring are also the serial steps of parallel processing, therefore, a MapReduce operation can also be used for processing.

The flow chart of the algorithm is shown in Figure 1. 


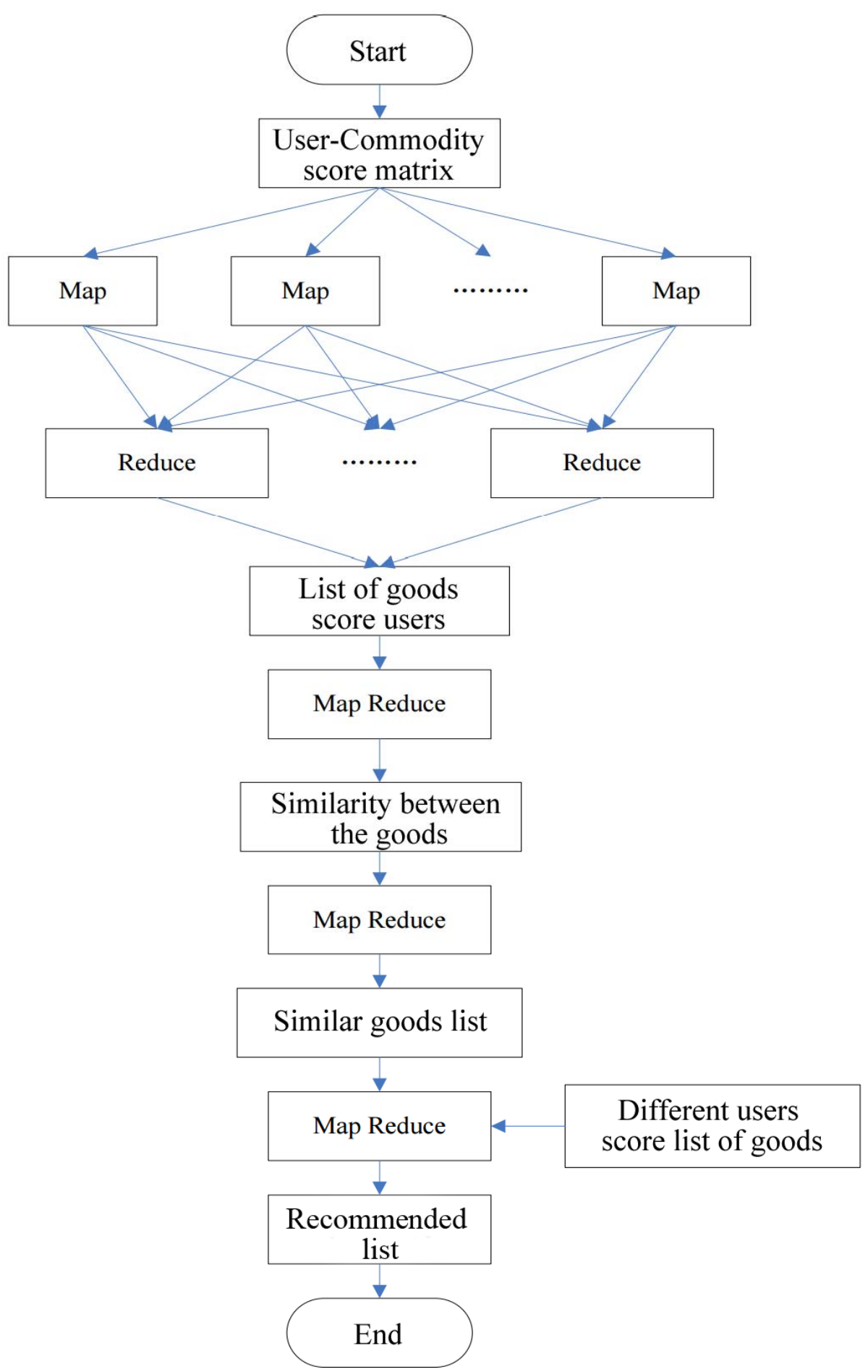

Figure 1 Flow chart of project-based collaborative filtering recommendation algorithm under cloud platform

Based on the above flow chart, the following project-based collaborative filtering recommendation algorithm step under cloud computing can be obtained:

Upload the user goods scoring to HDFS, and merge the score of each scored goods according to the user. 
Convert the key value between the user and goods to that of the same user's goods and goods, seek the different score on the same two goods from the same user, summarize all users' scores on these two goods, and calculate the similarity between two goods with the similarity formula.

Merge the similarity of each goods, and get the similarity list of each goods.

Predict user's score on the goods without evaluation, and return the recommendation result according to Top-N recommendation strategy.

\section{Parallelization of matrix decomposition-based ALS collaborative filtering recommendation algorithm under cloud platform}

The primary step for the parallelization of matrix decomposition- based ALS collaborative filtering recommendation algorithm is the parallelization of $U$ and $M$. The main time-consumption of the algorithm is the iteration for seeking the time of $U$ and $M$ multiplied by the number of iterations. According to the solving formula of $U$ and $M$, we can know that other user's feature vectors are not needed to seek the corresponding feature vector $U_{i}$ of user $\mathrm{i}$, there is no relationship in the solving process of the feature vector between users, and we just need the goods' feature matrix $\mathrm{M}$ and the evaluated goods collection from user $\mathrm{i}$; Other goods' feature vector is not needed when seeking the feature vector $V_{j}$ of goods $j$, there is no relationship in the solving process between goods, and we just need the user's feature matrix $\mathrm{U}$ and the collection of users evaluated the goods $\mathrm{j}$, so the solving process of $\mathrm{M}$ and $\mathrm{U}$ can be parallel handled.

\section{Conclusion}

The improved algorithm of two kinds of collaborative filtering recommendation algorithms are mainly introduced in the paper. The basic algorithm of two kinds of algorithms are introduced firstly, the principle for the algorithm parallelization to base on MapReduce is presented later. Combining the cloud computing and e-commerce personalized recommendation and applying in the e-commerce field via using the powerful storage capacity and efficient computing power in cloud computing can better improve user's shopping service quality, faster complete the e-commerce trade, and promote the win-win between the enterprise and the user.

\section{Reference}

[1] Walunj S G, Sadafale K. An online recommendation system for e-commerce based on apache mahout framework[C]//Proceedings of the 2013 annual conference on Computers and people research. ACM, 2013: 153-158.

[2] Chen S, Zhou L. Study on the Application of Intelligence Technology in the Warehouse[J]. Journal of Applied Science and Engineering Innovation, 2(8): 323-326.

[3] Zhao L, Hu N J, Zhang S Z. Algorithm design for personalization recommendation systems[J]. Journal of computer research and development, 2002, 39(8): 986-991.

[4] Li H, Liu H Y, Liu X G, et al. Resources Sharing Patterns Based on Cloud Computing[J]. Journal of Applied Science and Engineering Innovation, 2016, 3(5): 185-188. 